\title{
Design and simulation of A SIP-based Message Notification Mechanism
}

\author{
SONG Xiu-ping ${ }^{1, \text { a }}$ \\ ${ }^{1}$ Department of Communication Engineering CHONGQING COLLEGE OF ELECTRONIC \\ ENGINEERING, University city of Chongqing, China \\ aemai:sxp030210406@163.com
}

Keywords: event notification mechanism; message waiting indications; protocol extension; voice mail

\begin{abstract}
Session initiation protocol extension provides event status subscribe methods, which help to realization of event notification function. Message waiting indication is an application of event notification mechanism. There are many defects with using TCAP to realize MWI, and many terminals can not support MWI function. With development of intelligent terminals, MWI SIP-based gradually become the mainstream function. For many requirements of MWI function of terminals, article provides MWI model of SIP-based, and complete message simulation on intelligent network platform, deep analysis event notification system combine with instance, simulation outcome prove that SIP MWI is easy implementation and high efficiency.
\end{abstract}

\section{Introduction}

As event notification mechanism, Message Waiting Indications(MWI) are used to monitor change of mail status. Many terminals support MWI function. For instance, one small icon will appear in Android phones screen when receive MWI message. MWI functions including: type or number of message is changed, and then intelligent network will send MWI to terminal user when subscribe event notification service. MWI is applied to many fields, such as voice mail system and system mail notification and so on[1].

Realization MWI function can adopt many kinds of protocol, and usually use SS7 protocol (TCAP) to come true. But there are many defects with SS7 protocol. It is not easy to come true and many terminals can not support. Session initial protocol extension(RFC3265 (SIP)-Specific Event Notification) can solve this problem perfectly.

\section{Problems and solutions of TCAP MWI}

\section{Problems of TCAP MWI}

The following problems when use TCAP to realize MWI function:

(1)TCAP is used for center network structure with high complexity, and hard to come true;

(2)TCAP is almost to support old terminal without intelligence(traditional handset). And with bad supportive of intelligent, such as mobile phone with iOS, Android and windows phone8 and so on.

(3)Many functions are realized in network with TCAP, but event notification function are realized in terminal[2].

\section{Solutions of TCAP MWI}

To solve problem of realization MWI function with TCAP, this article raise one message model based session initial protocol extension: subscribe message trigger and status trigger mechanism, use SIP to realize event notification function replace TCAP. The following figure is SIP event model:

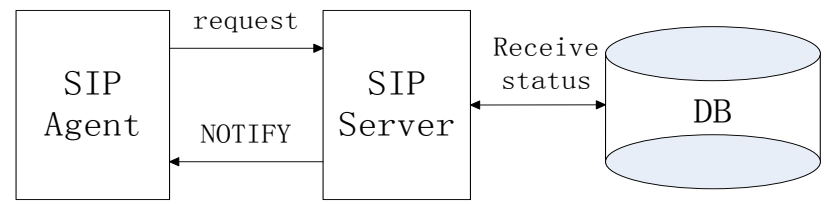

fig1. SIP event notification model 


\section{New Message Notification Model Design}

Message model based on SIP extension including two mechanisms: subscribe message trigger and status trigger.

\section{Session initial protocol extension}

There are three characters with easy extension: (1)message(method) extension;(2)message header extension;(3)message body extension. Event notification mechanism is formed by above extension. RFC3265 is emerged for this mechanism. Event notification mechanism have following concepts:(1)Event Package; (2)Notifier; (3)Subscriber; (4)Subscription[3].

\section{SUBSCRIBE message trigger}

Subscriber trigger effective subscribe event with SUBSCRIBE method, notifier send NOTIFY method with event status to Subscriber by select event status from database. Another scene: subscriber and notifier negotiation with each other, trigger event notification SUBSCRIBER with other messages, such as REGISTER method when terminal online. Figure 2 is event notification mechanism message flow.

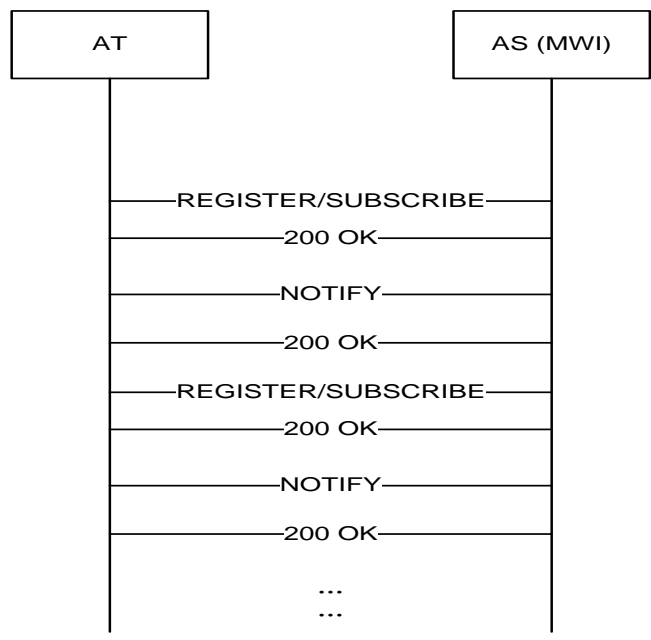

Fig2. Event notification mechanism message flow

\section{Status trigger}

If event status migration, and subscriber not initiate SUBSCRIBE method, then event status will not send to subscriber promptly. Notifier send event status to subscriber when defect change[4]. Then subscriber will receive status immediately. Figure 3 is MWI flow.

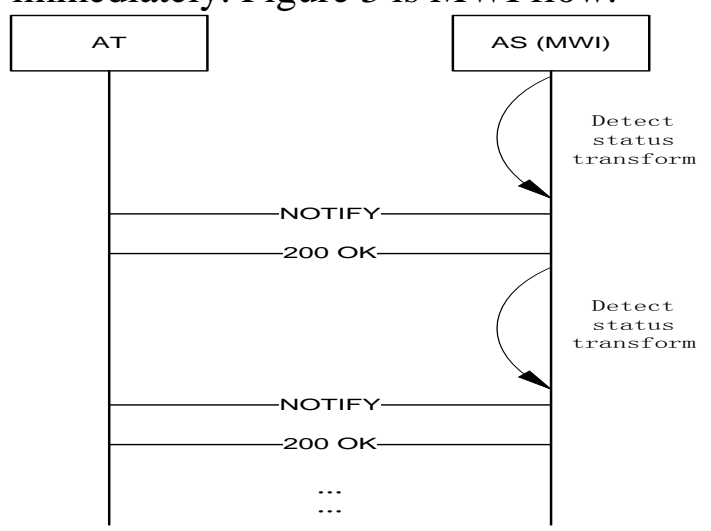

Fig3. MWI flow

\section{SIP MWI characteristics}

SIP MWI characteristics:

(1)SIP is pear to pear protocol, and support simple core network. Intelligent module is deploy at the network edge, embedded into the terminal. SIP is easy to come true and with high extensibility.

(2)SIP is mainstream signal protocol and support many terminals, especially for intelligent terminals. 
(3)Event notification function is come true at terminal, and SIP meet this requirement very well[5].

\section{Model Instance Analysis}

Applications of Event notification function: terminal status event, friends online status of instant message, change of message status and event notification of call status. Applied to many fields, especially for monitoring and security fields. This article will raise one application instance: message waiting indicator based on SIP, to realize the functions including: voice message, video message, fax message or email message and so on.

\section{System topology}

(1)Server send mail status by NOTIFY method after user terminal register successful;

(2)Server automatic send mail status by NOTIFY method to user terminal when new message arrival[6].

Figure 4 is Call flow:

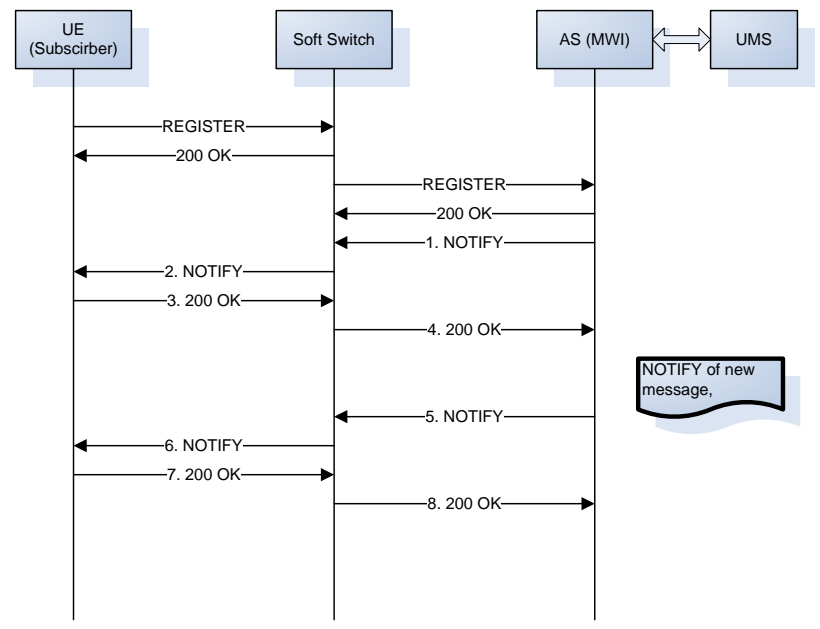

fig4. Call flow

\section{MWI application scenarios}

Voice mail service is one kinds of telecom value-added service, providing voice message sending and receiving for user based on voice mail system. Voice mail service is widely used in many operators. When receive new voice mail, system will sent new voice mail notification to alter user terminal, including MWI, text message, multimedia message and so on. The following is application scene of MWI in voice mail system.

(1) When call is unreachable, then transit to voice mail system to record voice. After record successful, called terminal will receive NOTIFY message when register, and can listen voice message;

(2) Called terminal send SUBSCRIBE method to select record message status;

(3) Called terminal will always received NOTIFY method until all messages are heard[7];

\section{Summary}

Event notification mechanism is consist of message header and message body extension by SUBSCRIBE and NOTIFY, which can be flexibly applied to services need get status information[8]. This article analysis in detail the design of MWI model of event notificaiton mechanism. Introduce messages trigger model, such as REGISTER and SUBSCRIBE message, which extend the application of MWI. It has very good reference value for new added-value services.

\section{References}

[1] Song XiuPing. Research and application of voice mail system based on SIP extension[J]. Master papaer,2013, 44-53. 
[2] Zhang Feng, Mi Zhengkun. SIP event notification extension mechanism[j], 2010, fifth period, 16-40.

[3] IETF RFC 3265. Session Initiation Protocol SIP Specific Event Notification [S]. 105-112.

[4] IETF RFC 3842.A Message Summary and Message Waiting Indication Event Package for the Session Initiation Protocol[S]. 4-16.

[5] IETF RFC 3261. SIP: Session Initiation Protocol [S]. 100-159.

[6] Yang Fan. Method for processing message wait notify service and access position register. CN100484280[P]. 2009.

[7] Wayne R.Howe, Jerome W. Schull. Visual message waiting indication in a telephone voice message system. US5363431[P].1994.

[8] Feng Jiangqiang. Development trend of Intelligent network[J]. China Telecom group Beijing research institute. 2011,101-120. 